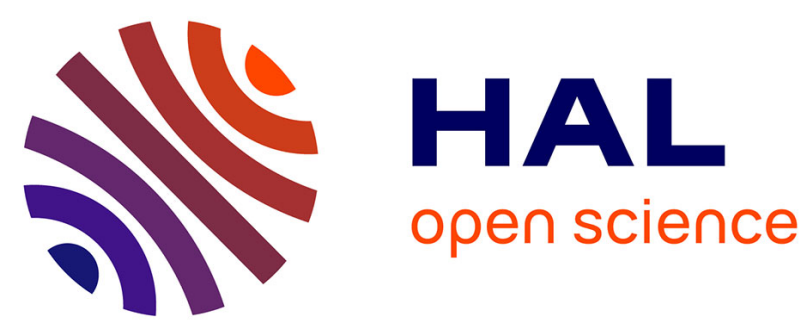

\title{
Détection de particules biologiques en suspension par ultrasons haute fréquence
}

\author{
Fabrice Lefebvre, Mohammadi Ouaftouh, Bertrand Nongaillard, Edouard \\ Radziszewski
}

\section{- To cite this version:}

Fabrice Lefebvre, Mohammadi Ouaftouh, Bertrand Nongaillard, Edouard Radziszewski. Détection de particules biologiques en suspension par ultrasons haute fréquence. Journal de Physique IV Proceedings, 1994, 04 (C5), pp.C5-1259-C5-1262. 10.1051/jp4:19945279 . jpa-00252970

\section{HAL Id: jpa-00252970 https://hal.science/jpa-00252970}

Submitted on 1 Jan 1994

HAL is a multi-disciplinary open access archive for the deposit and dissemination of scientific research documents, whether they are published or not. The documents may come from teaching and research institutions in France or abroad, or from public or private research centers.
L'archive ouverte pluridisciplinaire HAL, est destinée au dépôt et à la diffusion de documents scientifiques de niveau recherche, publiés ou non, émanant des établissements d'enseignement et de recherche français ou étrangers, des laboratoires publics ou privés. 


\title{
Détection de particules biologiques en suspension par ultrasons haute fréquence
}

\author{
F. LEFEBVRE, M. OUAFTOUH, B. NONGAILLARD et E. RADZISZEWSKI
}

Institut d'Electronique et de Micro-électronique du Nord, IEMN, Département

d'Opto-Acousto-Electronique DOAE, UMR 9929 du CNRS, Université de Valenciennes, BP. 311, Valenciennes cedex, France

\begin{abstract}
Résumé: Le comptage de particules constitue un domaine métrologique particulièrement important dans le secteur biomédical. L'utilisation des ondes acoustiques présente divers intérêts par rapport aux méthodes classiques tels que leur innocuité vis-à-vis des milieux biologiques, la possibilité d'examen de suspensions optiquement opaques,... Dans ce travail, nous nous proposons d'évaluer une méthode de détection de particules par absorption d'ultrasons haute fréquence (autour de $100 \mathrm{MHz}$ ). Les résultats expérimentaux obtenus sur une culture de protozoaires sont présentés et comparés à ceux fournis par la simulation du signal échographique provenant d'une structure multi-couches modélisant une cellule biologique. Le modèle théorique est basé sur le formalisme des matrices de transfert et prend en compte l'atténuation dans les différentes couches constituant la structure.

Abstract: In biomedical laboratories, an important part of work consists certainly in counting biological particles. The use of ultrasonic waves are particularly of interest because of their harmless towards biological tissues and their ability to control opacous suspension. In this paper, we will show that high frequency ultrasounds (about $100 \mathrm{MHz}$ ) are convenient for particles detection. The experimental results obtained with a culture of protozoa are compared to those given by simulation of echograms using matrix transfert formalism where the cell is asumed to be a multilayer structure in which the attenuation is taken into account in the different layers.
\end{abstract}

\section{INTRODUCTION.}

La plupart des compteurs de particules SPC (Single Particle Counters) utilisent un procédé de détection, soit optique (compteurs à obturation de lumière), soit électrique (compteurs de type COULTER) [1]. D'usage courant en laboratoire ou en industrie, ces compteurs ne permettent pas un certain nombre de contôles tels que le comptage dans les suspensions opaques pour les compteurs optiques ou introduisent une altération irrémédiable du milieu pour les compteurs du type COULTER. Nous avons présenté récemment une nouvelle technique de comptage utilisant des ondes acoustiques focalisées de haute fréquence qui permet de détecter des particules en suspension dans un liquide par réflexion des impulsions ultrasonores sur ces particules [2,3]. Avec ce système, le comptage s'effectue directement dans le milieu de culture sans qu'il soit nécessaire d'effectuer de prélèvement et sans danger pour les milieux biologiques. Un modèle linéaire de comptage, qui a été établi sur la base du bilan de transfert de particules à travers une surface, montre que l'information de concentration en particules dans le milieu peut être extraite du taux de comptages acoustiques par une simple relation de proportionnalité. Ce modèle a conduit à la réalisation d'un prototype de compteur qui nous a permis de vérifier cette proportionnalité sur divers milieux (sphèroïdes de latex, sang). Toutefois, dans le cas de certains types de milieux, le coefficient de réflexion 
sur les particules en suspension n'est pas suffisant pour garantir un rapport signal sur bruit acceptable pour la détection directe des échos dans la ligne RF. Cet état de fait nous a conduit à mettre au point une technique de détection par contraste de phase. Avant d'expliciter cette méthode, nous présenterons brièvement le contexte expérimental dans lequel nous nous plaçons. De façon à tester la faisabilité de cette méthode, nous avons simulé une cellule par une structure multi-couches et calculé le diagramme échographique en utilisant le formalisme des matrices de tranfert. Finalement, nous comparerons ces résultats théoriques à ceux obtenus par le système expérimental que nous avons réalisé.

\section{PRINCIPE DE BASE.}

L'objectif de ce travail, est la détection de cellules biologiques en mouvement dans une goutte de suspension placée entre un capteur acoustique focalisé et une lame de verre. Cette situation est représentée sur la figure 1 .

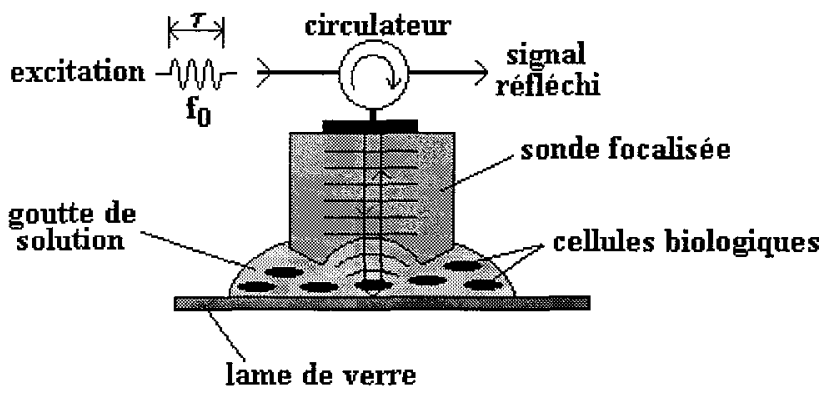

Fig. 1 - Principe de base de la détection de cellules.

Les cellules sont constituées d'une fine membrane de lipide d'environ $200 \mathrm{~nm}$ d'épaisseur contenant le cytoplasme de caractéristiques acoustiques proches de celles de l'eau. Le principe de base consiste à détecter les variations de phase dues au passage d'une cellule dans le foyer du capteur par rapport à la situation où aucune particule n'y est présente. Compte tenu des dimensions des cellules (environ $40 \mu \mathrm{m}$ ) comparativement à la largeur du foyer (environ $8 \mu \mathrm{m}$ ), nous admetterons que l'on peut modéliser le système par deux structures multi-couches dont la description est donnée sur la figure 2 .

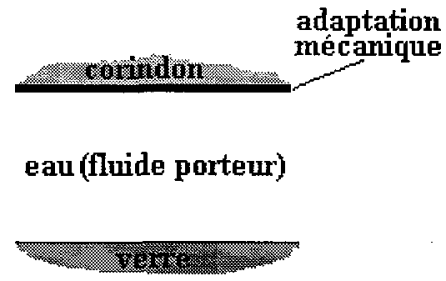

(A)

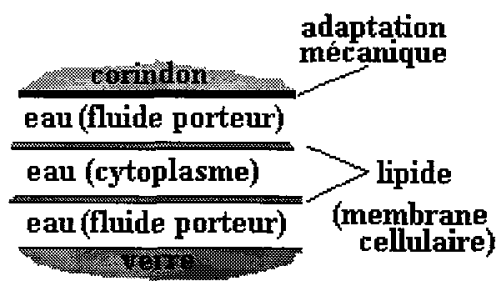

(B)

Fig. 2 - Modélisation par multi-couches des deux situations.

(A) absence de cellule dans la zone focale.

(B) présence d'une cellule dans la zone focale.

En l'absence de cellule, la structure est constituée par:

- deux substrats semi-infinis (en corindon représentant le capteur et en verre pour la lame)

- une couche d'adaptation mécanique en Trisulfure d'Arsenic

- une couche d'eau (fluide porteur)

Lorsqu'une cellule est présente dans le foyer, la structure est constituée par:

- deux substrats semi-infinis (en corindon représentant le capteur et en verre pour la lame) 
- une couche d'adaptation mécanique en Trisulfure d'Arsenic

- trois couches d'eau (fluide porteur et cytoplasme)

- deux couches de lipides (membranes cellulaires)

\section{SIMULATION DES STRUCTURES.}

Considérons une onde longitudinale arrivant sur une structure multi-couches telles que celles présentées sur la figure 2 ( $\mathrm{A}$ ou $\mathrm{B}$ ). Les milieux sont supposés homogènes, isotropes et atténuants (vecteur d'onde complexe). En utilisant la méthode des matrices de transfert [4], chaque couche peut être caractérisée par une matrice [P] qui relie les grandeurs mécaniques (déplacement $\mathbf{U}$ et contrainte $\mathbf{T}$ ) "de sortie" de la couche à celles "d'entrée". L'écriture des conditions aux limites sur les faces avant et arrière de la structure permet de calculer la réflectance sur la face avant:

$$
R=\frac{\left(\mathbf{p}_{21}+Z_{s} Z_{i} p_{12}\right)+j \cdot\left(Z_{s} p_{22}-Z_{i} p_{11}\right)}{\left(p_{21}-Z_{s} Z_{i} p_{12}\right)-j \cdot\left(Z_{s} p_{22}-Z_{i} p_{11}\right)}
$$

Où les $\mathbf{p}_{\mathbf{i j}}$ représentent les éléments de la matrice de passage globale de la structure, $\mathbf{Z}_{\mathbf{S}}$ l'impédance du substrat supérieur et $\mathbf{Z}_{\mathbf{i}}$ l'impédance du substrat inférieur. Dans le domaine de l'acoustique linéaire (faible puissance), les signaux réfléchi $\mathbf{s}(\mathbf{t})$ et incident $\mathbf{e}(\mathbf{t})$ sont liés par:

$$
s(t)=h(t) * e(t) \Leftrightarrow S(f)=H(f) . E(f)
$$

Où $\mathbf{h}(\mathbf{t})$ est la réponse impulsionnelle de la structure et $\mathbf{H}(\mathbf{f})$ sa réponse fréquencielle qui est identifiée à la réflectance $\mathbf{R}(\mathbf{f})$ dans le cas d'ondes planes. $\mathbf{S}(\mathbf{f})$ et $\mathbf{E}(\mathbf{f})$ sont respectivement les transformées de Fourier de $\mathbf{s}(\mathbf{t})$ et $\mathbf{e}(\mathbf{t})$. Pour la simulation, nous avons choisi un signal d'excitation constitué d'une sinusoïde pure à $100 \mathrm{MHz}$ modulée en amplitude par une impulsion de forme gaussienne.
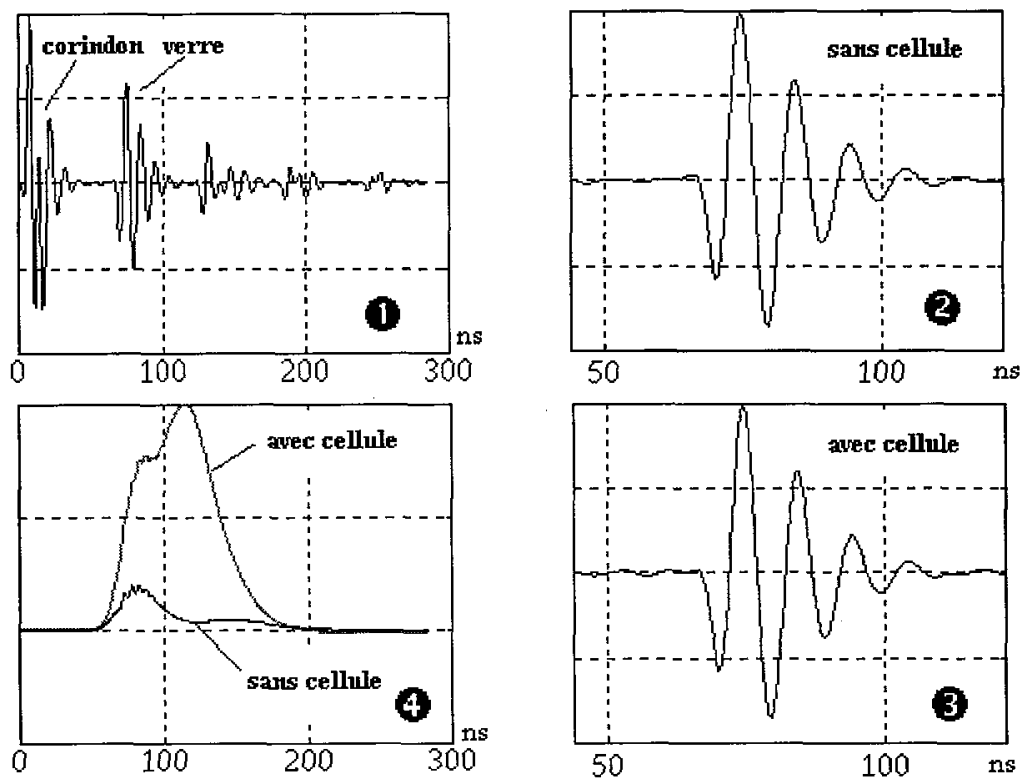

Fig. 3 - Résultats de simulation.

Le signal $\mathbf{s}(\mathbf{t})$ est calculé à partir de $\mathbf{S}(\mathbf{f})$ par transformée de Fourier inverse. La figure 3-1 montre le signal échographique complet. Les figures 3-2 et 3-3 représentent respectivement les échos sur la lame de verre 
en l'absence et en présence d'une cellule. Ces signaux montrent clairement qu'il n'y a pas de différence significative excepté une très légère variation de phase.

\section{EXPERIMENTATION.}

De façon à valider les résultats de simulation présentés au paragraphe précédent, nous avons réalisé le système de détection dont le schéma est donné sur la figure 4-1. Un oscillateur à boucle à vérouillage de phase délivre une porteuse très stable à $100 \mathrm{MHz}$ qui est séparée en deux parties par un diviseur de puissance. L'une est découpée par une impulsion rectangulaire de façon à produire le signal d'excitation. L'autre est utilisée pour le mélange avec l'écho réfléchi par la lame de verre préalablement amplifié et sélectionné par une porte retardée par rapport à l'émission. Après mélange et filtrage passe-bas, on obtient un signal contenant l'information de phase. En l'absence de cellule, la distance entre le capteur et le verre est ajustée de manière à annuler pratiquement le signal filtré. La figure 4-2 montre un très bon accord avec les résultats de simulation. Ce signal est ensuite comparé à un seuil afin de produire le signal logique de détection comme l'indique la figure 4-3. Celui-est ensuite dirigé vers une chaîne de comptage.

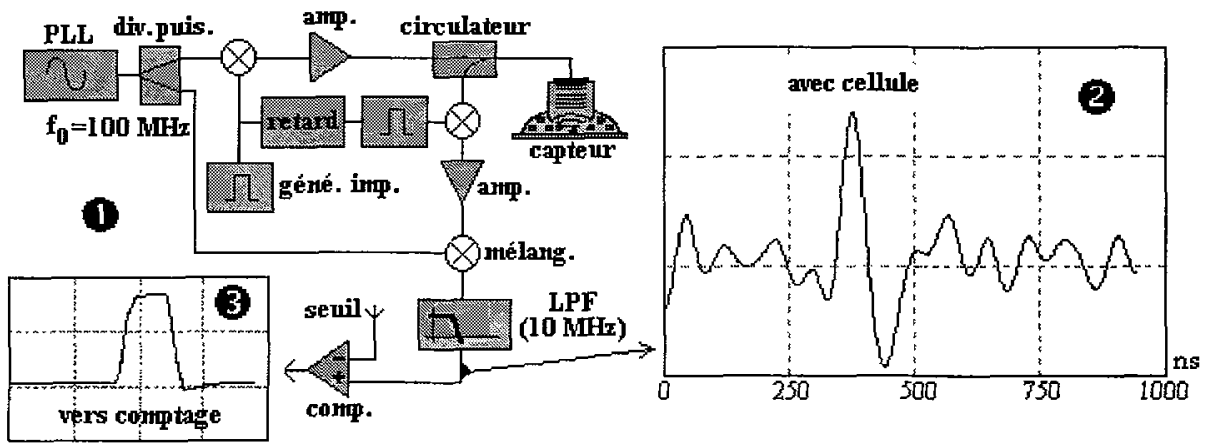

Fig. 4 - Le système de détection et les différents signaux expérimentaux.

\section{CONCLUSION.}

Dans cet article, nous avons utilisé la méthode de contraste de phase pour la détection de cellules biologiques en suspension dans une goutte placée sur une lame de verre. Nous avons montré la faisabilité de cette technique sourtout dans le cas où la détection des cellules par réflexion n'est pas envisageable. Les résultats obtenus expérimentalement et par simulation montrent une différence significative entre les signaux réfléchis, après traitement, dans les cas correspondant à la présence et l'absence d'une cellule. En associant une chaîne électronique de comptage à ce système, il est possible de déterminer la cencentration des cellules en suspension.

\section{REFERENCES}

[1]- T. Allen, "Particle size measurement", Powder technology series, Chapman and Hall, (1990).

[2]- F.Lefebvre, M. Toubal, E. Radziszewski, Y. Deblock, B. Nongaillard, Acoustical Imaging, Nanjing (1992), China.

[3]- F.Lefebvre, E. Radziszewski, Y. Deblock, M. Toubal, B. Nongaillard, Innovation et Technologie en Biologie et Médecine (1993), accepté pour publication.

[4]- L.M. Brekhovskikh, "Waves in layered media", Academic Press (1960), New-York. 